OPEN ACCESS

Edited by:

Christoph Hess,

University of Basel, Switzerland

Reviewed by: Robert Adam Harris, Karolinska Institutet, Sweden

Alessandra Mancino, Istituto Europeo di Oncologia, Italy

*Correspondence:

Sarah-Maria Fendt sarah-maria.fendt@vib-kuleuven.be; Jo A. Van Ginderachter jvangind@vub.ac.be

Specialty section: This article was submitted to Molecular Innate Immunity, a section of the journal

Frontiers in Immunology

Received: 23 December 2016 Accepted: 28 February 2017 Published: 15 March 2017

Citation:

Geeraerts X, Bolli E, Fendt S-M and Van Ginderachter JA (2017)

Macrophage Metabolism As Therapeutic Target for Cancer Atherosclerosis, and Obesity.

Front. Immunol. 8:289. doi: 10.3389/fimmu.2017.00289

\section{Macrophage Metabolism As Therapeutic Target for Cancer, Atherosclerosis, and Obesity}

\author{
Xenia Geeraerts ${ }^{1,2}$, Evangelia Bolli, ${ }^{1,2}$, Sarah-Maria Fendt ${ }^{3,4 *}$ and Jo A. Van Ginderachter, ${ }^{1,2 *}$ \\ ${ }^{1}$ Laboratory of Myeloid Cell Immunology, VIB Inflammation Research Center, VIB, Ghent, Belgium, ${ }^{2}$ Laboratory of Cellular \\ and Molecular Immunology, Vrije Universiteit Brussel, Brussels, Belgium, ${ }^{3}$ Laboratory of Cellular Metabolism and Metabolic \\ Regulation, VIB Center for Cancer Biology, VIB, Leuven, Belgium, ${ }^{4}$ Laboratory of Cellular Metabolism and Metabolic \\ Regulation, Department of Oncology, KU Leuven and Leuven Cancer Institute (LKI), Leuven, Belgium
}

Macrophages are not only essential components of innate immunity that contribute to host defense against infections, but also tumor growth and the maintenance of tissue homeostasis. An important feature of macrophages is their plasticity and ability to adopt diverse activation states in response to their microenvironment and in line with their functional requirements. Recent immunometabolism studies have shown that alterations in the metabolic profile of macrophages shape their activation state and function. For instance, to fulfill their respective functions lipopolysaccharides-induced pro-inflammatory macrophages and interleukin- 4 activated anti-inflammatory macrophages adopt a different metabolism. Thus, metabolic reprogramming of macrophages could become a therapeutic approach to treat diseases that have a high macrophage involvement, such as cancer. In the first part of this review, we will focus on the metabolic pathways altered in differentially activated macrophages and link their metabolic aspects to their pro- and anti-inflammatory phenotype. In the second part, we will discuss how macrophage metabolism is a promising target for therapeutic intervention in inflammatory diseases and cancer.

Keywords: M1-M2 macrophage polarization, tumor-associated macrophages, microenvironment, immunometabolism, metabolic reprogramming, metabolic therapy, cancer, inflammatory diseases

\section{INTRODUCTION}

Immunometabolism is a fast evolving field, which determines the metabolic machinery of immune cells and investigates how changing their metabolic phenotype affects immune cell function. It is known that the microenvironment shapes the metabolism of cells, which in turn contributes to their functionality. Environmental signals involved in metabolic regulation are cytokines, growth factors, oxygen levels, and nutrient availability. There is a growing evidence that immune cells in a specific microenvironment, such as inflamed tissue or tumors, reprogram their metabolic phenotype to fulfill cellular needs, such as survival, growth, and proliferation, or to carry out specific effector functions, such as phagocytosis and cytokine production. By changing the metabolism of immune cells, in particular, macrophages, it will be possible to modulate their function, which would be useful in diseases with a high macrophage commitment. Hence, understanding immune cell metabolism and its regulation will be essential to use metabolism as a therapeutic target to affect disease outcome (1-3). 
In this review, we provide an overview of the current knowledge concerning the metabolism of differentially activated macrophages. We will discuss recent findings on macrophage metabolism in the context of cancer and inflammatory diseases such as obesity and atherosclerosis. Furthermore, we will comment on promising metabolic targets for therapeutic purposes and approaches to reprogram macrophage metabolism in particular diseases.

\section{OVERVIEW ON THE MOST RELEVANT METABOLIC PATHWAYS IN MACROPHAGES}

Metabolism is a network of highly interconnected biochemical reactions required to generate metabolic products, such as energy and macromolecules from nutrients, provided by the microenvironment. Despite the plasticity of the metabolic network, immune cells, including macrophages, usually stick to a unique metabolic phenotype to accomplish their function. For example, while multiple ways [i.e., glycolysis and oxidative phosphorylation (OXPHOS)] exist to produce energy, macrophages generally prefer particular pathways for energy production in relation to their functional requirements.

A pathway that allows energy and biomass production is glycolysis. During glycolysis, extracellular glucose is taken up by the cell and subsequently converted to two molecules of pyruvate and ATP whereas $\mathrm{NAD}^{+}$is converted to NADH. To maintain glycolytic flux, cells can regenerate $\mathrm{NAD}^{+}$by converting pyruvate into lactate. Glycolysis also provides the intermediate glucose6-phosphate, which is the first molecule of the pentose phosphate pathway (PPP). The PPP consists of an oxidative branch that produces NADPH that is required to maintain the cellular redox balance and the production of fatty acids. The nonoxidative PPP branch provides intermediates used as precursors for nucleotide and amino acid synthesis. Although glycolysis is not the most efficient way to generate high amounts of ATP (two ATP molecules per glucose molecule), high glycolytic rates allow the cell to quickly produce sufficient energy and biosynthetic intermediates for cell growth and to fulfill its functional demands. For instance, the in vivo functions of macrophages not only encompass the insurance of tissue homeostasis under steady state but also a multitude of activities such as phagocytosis and cytokine production, upon activation (4). These functions have been referred to as SHIP: sample, heal, inhibit, and present (antigen) (5). Other cells that use glycolysis as major pathway for biomass production are cancer cells (6). Already in the early twentieth century, Otto Warburg postulated that cancer cells preferentially convert glucose into lactate, even in the presence of oxygen, a process better known as the Warburg effect (7). On the other hand, in the presence of oxygen, cells usually produce ATP via the electron transport chain (ETC) which is coupled to the tricarboxylic acid (TCA) cycle. Acetyl coenzyme A (acetyl-CoA) usually serves as the entry point of glycolytic carbon into the TCA cycle. The reducing equivalents $\mathrm{NADH}$ and $\mathrm{FADH}_{2}$, generated by the TCA cycle, serve as electron carriers that transfer electrons through ETC for OXPHOS, an oxygen-driven process that produces high amounts of ATP (theoretically up to 36 ATP molecules per glucose molecule). Moreover, cells can use different carbon sources, such as glutamine or fatty acids, to fuel into the TCA cycle. While glutamine can be converted into the TCA cycle intermediate $\alpha$-ketoglutarate, the fatty acid oxidation pathway (FAO, also known as $\beta$-oxidation) degrades fatty acids into acetyl-CoA, $\mathrm{NADH}$, and $\mathrm{FADH}_{2}$, which are further used to produce ATP. In general, OXPHOS is a highly efficient way for ATP production, preferred by cells with high energy demands or cells that require longevity to function over a long period of time $(3,8)$.

Besides energy production, intermediates from different metabolic pathways, such as glycolysis, PPP, and the TCA cycle can be used as precursors for de novo synthesis of nucleotides, fatty acids, and amino acids, which are essential building blocks for the cell. This requires increased replenishment of metabolic pathway intermediates via anaplerotic reactions. A well-known example is the replenishment of TCA cycle intermediates via direct conversion of pyruvate into oxaloacetate (OAA) by pyruvate carboxylase, production of $\alpha$-ketoglutarate from glutamate or the conversion of adenylosuccinate into fumarate.

\section{METABOLIC SIGNATURE OF MACROPHAGES}

\section{Macrophage Activation States}

Macrophages are considered as polyvalent cells in our body, playing a key role during embryonic development and contributing to tissue repair and inflammation (9). As a consequence, macrophages have a high plasticity and are able to adapt their phenotype, as instructed by their microenvironment and in agreement with their functional requirements (10). This has resulted in a spectrum model of macrophage activation, illustrating the divergent transcriptome of macrophages exposed to a broad variety of cues (11). Furthermore, this has led to a new proposal of nomenclature, whereby the triggers that determine the macrophage's phenotype are specified (12). Additionally, other nomenclatures have been proposed to distinguish macrophage populations based on their function and encompassing both in vitro and in vivo situations (13). Despite the diversity of signals they can be subjected to and the different proposals for classifying them, macrophages for a long time have been classified in two main groups, representing the extremes of a continuum, namely "classically activated" or M1 and "alternatively activated" or M2 macrophages (14-16). Upon stimulation with interferon- $\gamma$ (IFN $\gamma$ ) and toll-like receptor (TLR) ligands, such as lipopolysaccharides (LPS), macrophages obtain a pronounced pro-inflammatory M1 phenotype, characterized by the secretion of pro-inflammatory cytokines and reactive nitrogen and oxygen species. Furthermore, M1 macrophages possess bactericidal and antitumor activity. Conversely, the Th2 cytokines interleukin (IL)-4 and IL-13 polarize macrophages toward an anti-inflammatory M2 phenotype. Alternatively, activated macrophages are involved in tissue remodeling, immunosuppression, and show phagocytic and protumoral activity $(12,17)$. Although this dichotomous M1-M2 model is an oversimplification that only represents two extremes in a spectrum of macrophage activation states, it has been found that under pathological conditions, 
macrophages in vivo regularly mimic these two polarization states. Moreover, the M1/M2 nomenclature has been extensively used in multiple papers that are being referenced here, justifying the use of this nomenclature throughout this review. Imposed by their microenvironment, macrophages adapt their metabolic phenotype to fulfill their function in homeostasis and inflammation (18).

Briefly, the metabolism of M1 macrophages is characterized by enhanced glycolysis, flux through the PPP, fatty acid synthesis, and a truncated TCA cycle, leading to accumulation of succinate and citrate. The metabolic profile of M2 macrophages is defined by OXPHOS, FAO, a decreased glycolysis, and PPP (19). In the next section, we will discuss in more detail the metabolic signature of M1 and M2 macrophages and focus on the link between metabolism and macrophage functionality.

\section{M1 Macrophage Metabolism Is Characterized by Aerobic Glycolysis, Fatty Acid Synthesis, and a Truncated TCA Cycle}

Integrated transcriptional and metabolic network analysis revealed that activation of macrophages by IFN $\gamma$ and LPS gives rise to a TCA cycle that is truncated at the level of isocitrate dehydrogenase (IDH) and succinate dehydrogenase (SDH) leading to the accumulation of succinate and citrate metabolites (Figure 1). The build-up of citrate is the result of transcriptional downregulation of IDH1, the enzyme responsible for the conversion of isocitrate (an isomer of citrate) into $\alpha$-ketoglutarate. The accumulated citrate serves as a precursor for the synthesis of the macrophage-specific metabolite itaconic acid, which is a major feature of IFN $\gamma /$ LPS-polarized macrophages. Further supporting this phenomenon, Jha and colleagues found that immunoresponsive gene 1 (irg1) is one of the most upregulated genes in IFN $\gamma /$ LPS-treated macrophages. Irg1 codes for the enzyme cis-aconitate decarboxylase that converts aconitate (derived from citrate) to itaconic acid (20). To ensure carbon entry into the truncated TCA cycle, LPS-activated macrophages repress pyruvate dehydrogenase kinase 1 , which in turn leads to sustained conversion of pyruvate into acetyl-CoA via pyruvate dehydrogenase (21).

A study in murine and human macrophages revealed an antimicrobial effect of itaconic acid against Mycobacterium tuberculosis and Salmonella enterica, most likely by inhibition of the bacterial glyxoylate shunt pathway enzyme isocitrate lyase. Hence, itaconic acid is a first example of how cellular metabolism is linked to the antimicrobial function of pro-inflammatory macrophages (22). Recently, itaconic acid has been put forward as a specific driver for succinate accumulation in LPS-stimulated macrophages $(23,24)$, since it inhibits SDH (Figure 1). Hence, itaconic acid explains the second truncation in the TCA cycle of IFN $\gamma /$ LPS-treated macrophages. In accordance, it was shown that LPS-induced bone marrow-derived macrophages from irg $1^{-/-}$mice did not display succinate accumulation because of impaired itaconic acid production (23). Different mechanisms have been proposed to explain carbon flow to succinate within a dysfunctional TCA cycle. One of them is glutamine-dependent anaplerosis through $\alpha$-ketoglutarate or glutamine-derived succinate replenishment via the GABA shunt pathway. Either way, LPS-stimulated macrophages showed high levels of the glutamine transporter Slc3a2 (25).

Metabolite concentration changes can directly alter the activity of signaling pathways (26). The accumulation of succinate in LPS-stimulated macrophages is an example of this regulation via metabolite concentrations, since it stabilizes HIF- $1 \alpha$ by limiting prolyl hydroxylase domain activity (Figure 1). In turn, HIF-1 $\alpha$ stabilization induces the expression of the pro-inflammatory cytokine IL-1 $\beta$ (25). Additionally, a recent study proposes that succinate may indirectly stabilize HIF- $1 \alpha$ via the induction of reactive oxygen species (ROS) (27). In any case, mature IL-1 $\beta$ production requires inflammasome activation, which supports pro-IL-1 $\beta$ to IL-1 $\beta$ processing (4). Interestingly, also inflammasome activation may be regulated by metabolic cues, as demonstrated by the finding that mTORC1-induced hexokinase (HK)-1-dependent glycolysis activates the NLRP3 inflammasome in macrophages upon LPS activation (28).

Furthermore, the stabilization of HIF- $1 \alpha$ by succinate can be linked to an increased glycolytic flux (Figure 1), which is another metabolic signature of classically activated M1 macrophages, considering that HIF induces several glycolytic genes, such as glucose transporter 1 (GLUT1) (29), PFKFB3 (30), and monocarboxylate transporter 4 (MCT4) (31). The fact that LPS is able to induce hypoxic gene expression in macrophages in the absence of hypoxia has been suggested before (32). The expression of GLUT1 in M1 macrophages emphasizes the importance of glucose as carbon source for pro-inflammatory macrophages. Freemerman and colleagues demonstrated that overexpression of GLUT1 in the murine macrophage cell line RAW264.7 induces an M1 phenotype, characterized by the expression of inflammatory mediators and the production of ROS (33). Another HIF- $1 \alpha$ regulated gene is PFKFB3, which codes for the ubiquitous 6-phosphofructo-2-kinase/fructose-2,6-bisposphatase isoform (U-PFK2). The expression of U-PFK2 is considered as an underlying mechanism of a high glycolytic flux in M1 macrophages. In particular, upon IFN $\gamma / \mathrm{LPS}$ stimulation, the expression of PFK2 shifts from the liver isoform (L-PFK2) to the more active ubiquitous isoform (U-PFK2). U-PFK2 converts fructose-6-phosphate to fructose-2,6-biphosphate that in turn activates the glycolytic enzyme phosphofructo-1-kinase (PFK1) and consequently increases glycolytic flux $(34,35)$ because of its dominant kinase activity. Since the TCA cycle is truncated in M1 macrophages, lactate production regenerates the majority of the $\mathrm{NAD}^{+}$needed to sustain glycolysis. Consequently, lactate is exported to the extracellular environment by (MCT4). Data indicate that knocking down MCT4 in LPS-induced macrophages leads to enhanced accumulation of intracellular lactate, a decrease in the expression of the glycolytic enzymes HK-2 and PFKFB3 and a reduction of the pro-inflammatory cytokines IL- 6 and tumor necrosis factor (TNF) $\alpha(36)$.

Another aspect of M1 macrophages is an enhanced PPP (25), which is not unexpected considering the high glycolytic flux. The PPP generates NADPH, which is required as a cofactor for LPS-induced inducible nitric oxide synthase (iNOS) to catabolize arginine into nitric oxide (NO) and L-citrulline (Figure 1). To sustain antimicrobial NO production, imported L-citrulline can 


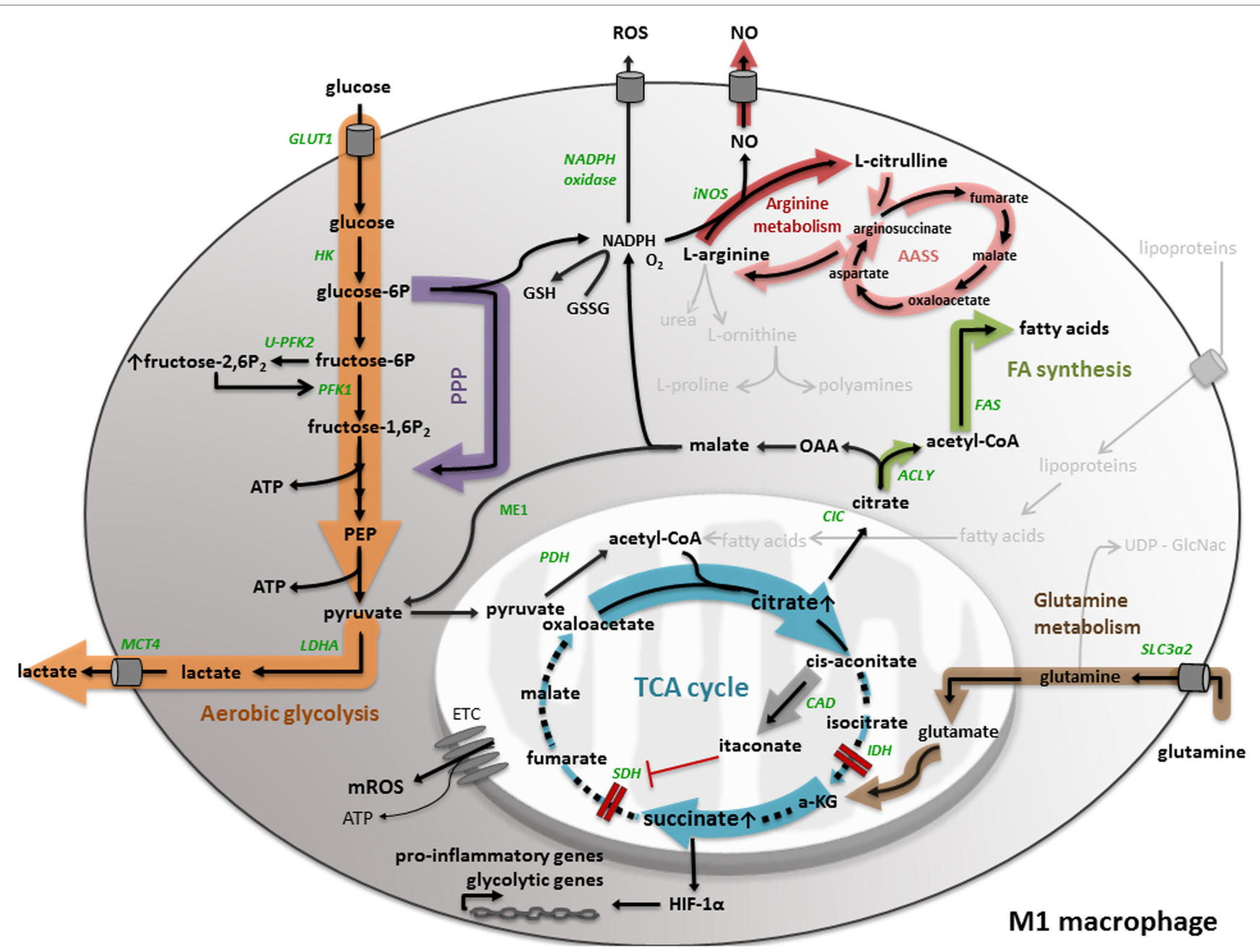

FIGURE 1 | M1 macrophage metabolism. M1 macrophage metabolism is characterized by enhanced aerobic glycolysis, converting glucose into lactate. M1 macrophages have an increased flux through the pentose phosphate pathway (PPP), generating NADPH, used for the generation of the anti-oxidant glutathione (GSH) and the inflammatory mediators nitric oxide (NO) and reactive oxygen species (ROS). In M1 macrophages, the tricarboxylic acid (TCA) cycle is broken in two places, leading to the accumulation of succinate and citrate. While the accumulation of succinate leads to HIF-1 $\alpha$ stabilization and the transcription of proinflammatory and glycolytic genes, citrate is used for the generation of fatty acids, NO, ROS, and the synthesis of itaconate. Another aspect of M1 macrophage metabolism is the conversion of L-arginine to $\mathrm{NO}$ and L-citrulline. All important metabolic reactions present in M1/M2 macrophages are shown in black, reactions shown in gray are absent or less pronounced. The metabolic pathways strongly upregulated by M1/M2 macrophage polarization are highlighted by a colored shadow, the width of the shadow illustrates the weight of a particular pathway in the macrophage activation state. All metabolic enzymes are indicated in green. Dotted lines represent impaired metabolic reactions. Abbreviations: $\alpha$-KG: $\alpha$-ketoglutarate; AASS: aspartate-arginosuccinate shunt pathway; ACLY: ATP-citrate lyase; CAD: cis-aconitate decarboxylase; CIC: mitochondrial citrate carrier; ETC: electron transport chain; FAS: fatty acid synthase; GLUT: glucose transporter; HK: hexokinase; IDH: isocitrate dehydrogenase; iNOS: inducible nitric oxide synthase; LDH: lactate dehydrogenase; MCT: monocarboxylate transporter; ME: malic enzyme; OAA: oxaloacetate; PEP: phosphoenolpyruvate; PDH: pyruvate dehydrogenase; PFK: phosphofructokinase; SDH: succinate dehydrogenase; SLC: solute carrier.

be recycled to arginine by the so-called aspartate-arginosuccinate shunt pathway (AASS) (37). NO is not only an antimicrobial agent, but is also put forward as a key regulator of M1 macrophage metabolism. NO actually causes nitrosylation of the iron-sulfur-containing ETC complexes and consequently inhibits mitochondrial respiration and $\operatorname{OXPHOS}(38,39)$. Recent research by Van den Bossche and colleagues demonstrated that disturbed mitochondrial OXPHOS, caused by IFN $\gamma / \mathrm{LPS}$ induced NO production, can prevent M1 to M2 polarization after IL-4 stimulation (40). Furthermore, PPP-produced NADPH can be used as a cofactor for NADPH oxidase, which is involved in the generation of ROS (41). In general, the NADPH-driven production of the inflammatory mediators NO and ROS once again indicates the tight link between M1 metabolism and its antimicrobial functionality. Nevertheless, TLR1/2/4 signaling in macrophages induces mitochondrial ROS production, despite increased NADPH production via the PPP, which also contributes to the bactericidal activity of macrophages (42).

An additional concept that is related to M1 macrophage metabolism is maintenance of the cellular redox balance. 
NADPH can be used to generate the anti-oxidant glutathione (Figure 1), which is essential to maintain redox homeostasis and prevent cellular damage by ROS (8). NAPDH can be produced by the PPP, and also citrate can be exported to the cytosol where it contributes to NADPH production and regulation of the redox balance. In particular, the mitochondrial citrate carrier (CIC), encoded by the Slc25al gene, exports citrate from the mitochondria to the cytosol where it is cleaved back to acetyl-CoA and OAA by the enzyme ATP-citrate lyase (ACLY). In macrophages, the expression of both CIC and ACLY is induced by inflammatory stimuli, such as LPS, TNF $\alpha$, and IFN $\gamma$ through NF-kB and/or signal transducer and activator of transcription (STAT) signaling (43-45). Within the cytosol, OAA can be reduced to malate, which is converted to pyruvate by malic enzyme (ME1) with production of NADPH. However, in their network analysis, Jha and colleagues did not detect ME1 activity in IFN $\gamma /$ LPS-induced macrophages (20). Taken together, citrate-derived NADPH can be used, as described previously, for NO and ROS production and might support the redox balance (43-45), which again associates a metabolic intermediate to the pro-inflammatory functionality of M1 macrophages.

Moreover, it has been shown in several studies that specific metabolites, such as acetyl-CoA, can regulate the activation of chromatin-modifying enzymes, making the link between metabolism and epigenetics. For example, citrate-derived acetyl-CoA can be used for histone acetylation (46). Especially the expression of some glycolytic genes, such as HK-2, $\mathrm{PFK} 1$, and lactate dehydrogenase-A is regulated in an ACLYdependent manner and thus subjected to histone acetylation (47). Besides, acetyl-CoA can be used for the biosynthesis of fatty acids (Figure 1), a last aspect of M1 macrophage metabolism we want to discuss. It was demonstrated before that LPS activation of TLR4 in macrophages induces lipid accumulation through several mechanisms, among which enhanced fatty acid uptake by the fatty acid transporter CD36, increased triglyceride synthesis, and diminished triglyceride lipolysis (48). Moreover, it was found that upon M-CSF mediated monocyte to macrophage differentiation, lipid synthesis increased through upregulation of the nuclear transcription factor sterol regulatory element-binding protein-1c, inducing expression of fatty acid synthesis-related genes, such as fatty acid synthase (49). Moreover, fatty acids are used as precursor for prostaglandin production in macrophages stimulated by TNF $\alpha$, LPS, or IFN $\gamma$ (43-45).

Since citrate is involved in several mechanisms that shape the pro-inflammatory phenotype of M1 macrophages, such as production of NO, ROS, NAPDH, itaconic acid, fatty acids, and histone acetylation, M1 macrophages developed mechanisms to ensure the preservation of high citrate levels. As described before, IFN $\gamma /$ LPS-treated macrophages show an upregulation of the AASS pathway, which connects the NO and TCA cycle. This pathway not only sustains NO production, but also replenishes citrate in the broken TCA cycle by anaplerosis (20).

Taken together, the above-described metabolic mechanisms of M1 macrophages contribute in a specific manner to their pro-inflammatory phenotype, highlighting the link between macrophage metabolism and functionality.

\section{The Metabolic Signature of M2 Macrophages Is Characterized by FAO, and an Oxidative TCA Cycle}

The metabolic phenotype of M2 macrophages shows significant differences with M1 macrophages, which is comprehensible regarding their differential function as anti-inflammatory component and mediator of tissue homeostasis (19).

One of the major metabolic differences between M1 and M2 macrophages is their energy metabolism. While M1 macrophages preferentially obtain their energy from glycolysis, M2 macrophages mainly produce ATP through an oxidative TCA cycle coupled to OXPHOS (Figure 2) (50). To fuel an oxidative TCA cycle, IL-4-stimulated macrophages rely on FAO (also known as $\beta$-oxidation) (51) and glutamine metabolism (20). In IL-4-induced macrophages, the important sources of fatty acids are triacylglycerol-rich lipoproteins, such as LDL and VLDL. These are taken up by the scavenger receptor CD36 and catabolized in the lysosome by lysosomal acid lipase (LAL). Studies in CD36- and LAL-deficient mice indicated that CD36-mediated lysosomal lipolysis of lipoproteins is essential for proper M2 activation (52). Another study demonstrated that expression of a permanently active mutant of carnitine palmitoyl transferase (CPT)-1a, which is essential for the transport of long-chain fatty acids along the mitochondria, enhanced FAO in macrophages, and reduced inflammation (53). However, a recent study indicated that macrophage-specific CPT2 depletion inhibits FAO, while not impairing M2 polarization after IL-4 stimulation (54). These data suggest that CPT1 might have an extra function in M2 polarization, independent of FAO (4). As mentioned before, glutamine can fuel the TCA cycle via anaplerotic generation of $\alpha$-ketoglutarate. Furthermore, glutamine contributes to UDPGlcNAc synthesis via the hexosamine pathway. UDP-GlcNAc leads to protein glycosylation, including the M2 markers macrophage mannose receptor (MMR) and macrophage galactose binding lectin (Mgl1) (20).

In IL-4/IL-13-stimulated macrophages, the upregulation of FAO and mitochondrial biogenesis is orchestrated by the combined action of STAT6, peroxisome proliferator-activated receptors (PPARs), and PGC-1 $\beta$ (55). Stimulation with Th2 cytokines IL-4 and IL-13 induces a cytoplasmic signaling cascade, resulting in activation of the transcription factor STAT6, which in turn induces expression of PPAR $\delta, \operatorname{PPAR} \gamma$, and the coactivator protein PGC-1 $\beta(55,56)$. Additionally, the expression of M2 markers, such as MMR, is regulated by the interplay of these components (57). Accordingly, the knockdown of PGC-1 $\beta$ was shown to decrease FAO upon IL-4 stimulation and impaired the suppression of pro-inflammatory cytokine production by IL-4, which underlines the importance of PGC- $1 \beta$ to sustain the antiinflammatory phenotype of M2 macrophages (51).

Besides differences in the energy metabolism, M1 and M2 macrophages show an opposing arginine metabolism (Figure 2), which is correlated to their functional polarization. While M1 macrophages upregulate iNOS and metabolize $\mathrm{L}$-arginine to the antimicrobial agent $\mathrm{NO}$ and L-citrulline, M2 macrophages catalyze L-arginine to urea and L-ornithine by inducing arginase (ARG-1). L-ornithine serves as precursor 


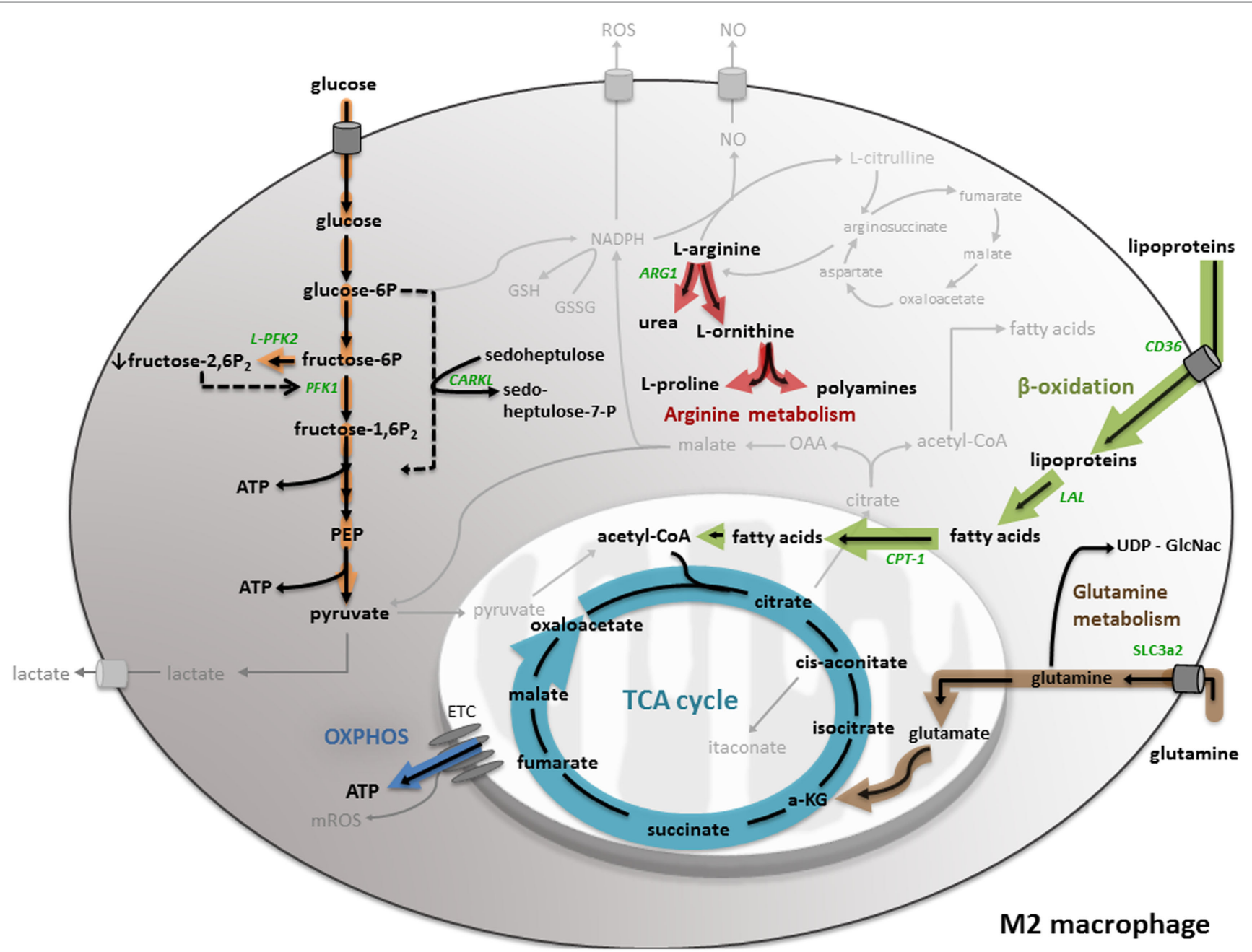

FIGURE 2 | M2 macrophage metabolism. M2 macrophages mainly produce ATP through an oxidative TCA cycle coupled to oxidative phosphorylation (OXPHOS). To fuel the TCA cycle, M2 macrophages rely on fatty acid oxidation (or $\beta$-oxidation) and glutamine metabolism. Furthermore, M2 macrophages show a lowered glycolysis and pentose phosphate pathway (PPP). Moreover, M2 macrophages convert L-arginine into urea and L-ornithine, which serves as precursor for L-proline production. All important metabolic reactions present in M1/M2 macrophages are shown in black, reactions shown in gray are absent or less pronounced. The metabolic pathways strongly upregulated by M1/M2 macrophage polarization are highlighted in orange. All metabolic enzymes are indicated in green. Dotted lines represent impaired metabolic reactions. Abbreviations: $\alpha$-KG: $\alpha$-ketoglutarate; ARG: arginase; CD: cluster of differentiation; CPT: carnitine palmitoyl transferase; ETC: electron transport chain; LAL: Iysosomal acid lipase; PEP: phosphoenolpyruvate; PFK: phosphofructokinase; SLC: solute carrier.

for L-proline production, which is used for collagen synthesis and accordingly contributes to wound repair, a key function of M2 macrophages (58). The expression of ARG-1 is induced by the Th2 cytokines IL- 4 and IL-10 (59) via activation of the transcription factor STAT6 $(60,61)$. Since NO is no longer produced in M2 macrophages, it cannot block the ETC and subsequently enables OXPHOS (40), pointing out the strong interconnection within the metabolic network of M2 macrophages. A recent study by Ref. (62) stated that ornithine decarboxylase (ODC), involved in polyamine metabolism, directly regulates macrophage activation. The authors showed that ODC-deficiency in macrophages alters histone modifications and changes the chromatine structure, leading to up-regulated transcription of M1 genes and increased inflammation during bacterial infections with Helicobacter pylori and Citrobacter rodentium (62).

In contrast to M1 macrophages, M2 macrophages show a lowered glycolysis (Figure 2). IL-4/IL-13-stimulated macrophages express the L-PFK2 isoform, encoded by the PFKFB1 gene, which has a dominant biphosphatase activity, resulting in low levels of the glycolytic activator fructose2,6-biphosphate (34). Correlated to low glycolytic rates, M2 macrophages have a limited flux through the PPP. IL-4/ IL-13 stimulation promotes the expression of kinase-like protein (CARKL), a sedoheptulose kinase that catalyzes the production of sedoheptulose-7-phosphate and limits PPP flux in a not fully understood manner. Moreover, overexpression of CARKL in macrophages leads to decreased production 
of pro-inflammatory cytokines, which is consistent with the M2 phenotype (63).

\section{MACROPHAGE METABOLISM AS A PROMISING TARGET FOR THERAPEUTIC INTERVENTION IN CANCER AND OTHER INFLAMMATORY DISEASES}

In multiple inflammatory diseases, macrophages are leading cells affecting disease outcome. For example, a tumor is considered as a nonhomogeneous mass wherein a complex interaction exists between cancer cells and tumor-infiltrating immune cells, such as macrophages. These so-called tumor-associated macrophages (TAM) are one of the most prominent immune cells in a tumor. Although macrophages can target transformed cells, cancer cells evade this defense by reprogramming macrophages so that they support tumor progression $(17,64)$. The cancer context is a good example where metabolic targeting is already assessed as therapeutic target. Cancer cells have, as a consequence of their highly proliferative potential, a metabolic signature that is notably different compared to nonmalignant cells. Several drugs that specifically target the metabolic pathways of cancer cells are in clinical trials $(65,66)$. However, in other inflammatory diseases, such as obesity and atherosclerosis, macrophages are highly involved in disease progression. Hence, macrophages are an intriguing target for therapy in several disorders. In the next section, we will discuss recent discoveries concerning macrophage metabolism in cancer and inflammatory diseases and we will put forward interesting strategies for therapeutic intervention.

\section{Cancer: Repolarizing Macrophages toward a Pro-inflammatory Phenotype}

Tumor-associated macrophages are amongst the most abundant inflammatory cells in tumors and a significant correlation was found between high TAM density and a worse prognosis for most cancers (67). By now, it is clear that TAM exert several tumorpromoting functions, including stimulation of angiogenesis, remodeling of the extracellular matrix, promotion of cancer cell survival, proliferation, invasion, extravasation and metastasis, and suppression of antitumor immunity (68). Within the same tumor, the co-existence of two distinct TAM subpopulations has been shown, both derived from tumor-infiltrating inflammatory monocytes: M2-like MHC-II ${ }^{\text {low }}$ TAM that reside in the hypoxic regions of the tumor and perform angiogenic, immunosuppressive and protumoral activities and M1-like MHC-II ${ }^{\text {high }}$ TAM that are present in the normoxic tumor regions and possess pro-inflammatory and antitumoral characteristics. Importantly, this dichotomous TAM phenotype remained valid in several independent transplantable and transgenic mouse tumor models (69-71). Studies indicate that the TAM phenotype depends on the stage of tumor development and that the majority of TAM in late stage tumors is M2-like (72) (Figure 3). M2-like TAM highly stimulate tumor progression and have been shown to affect the efficacy of anticancer treatments, contribute to therapy resistance, and mediate tumor relapse following conventional cancer therapy (9). Therefore, intervening with M2 TAM functionality is a plausible avenue for the development of new immunotherapies.

Before, TAM-targeted antitumor strategies were mainly based on the inhibition of macrophage recruitment to the tumor and suppression of TAM survival (73). For example, CCL2 blocking agents, which prevent monocyte recruitment to the tumor and subsequent generation of monocyte-derived TAM, have been shown to impair tumor progression in several tumor models (74-76). As a cautionary note, a recent study in breast carcinoma demonstrated that interruption of CCL2 inhibition was associated with increased cancer cell mobility and blood vessel formation, leading to accelerated metastasis and cancer death (77). Hence, ablation of TAM as a monotherapy might be insufficient for prolonged tumor control. Furthermore, general macrophage depletion could cause side effects, such as increased susceptibility for infections (78).

Therefore, strategies that reprogram protumoral M2 TAM into an antitumoral M1 phenotype, without depleting the full TAM population, gained attention and are currently highly investigated. In this context, Casazza and colleagues showed that genetic depletion of neuropilin-1 in TAM prevents the entry of TAM in the hypoxic regions of the tumor. TAM that accumulate in the normoxic tumor area adopt a more M1-like phenotype, which initiates a cascade of antitumor immunity, leading to reduced angiogenesis, tumor growth, and metastasis (79). Recent research indicated that blocking M-CSFR signaling impaired the differentiation of tumor-infiltrating monocytes into MHC-II ${ }^{\text {low }}$ M2-like TAM and consequently shifted the M1/M2 TAM balance toward the antitumoral TAM phenotype (71). Additionally, studies in glioblastoma illustrated that M-CSFR inhibition impaired the M2 phenotype and the tumor-promoting functions of TAM, without affecting TAM numbers (80).

Since macrophage metabolism is inextricably connected to its functionality, metabolic reprogramming of M2-like TAM might be an elegant way to repolarize TAM toward an antitumoral phenotype and thus affect tumor growth and metastasis. Although studies about TAM metabolism are rather limited at this moment, there is an emerging evidence that unraveling the TAM phenotype might lead to the identification of alternative, novel targets for TAM-directed intervention.

A study concerning TAM metabolism by Colegio and colleagues indicated that tumor-derived lactate is necessary to polarize TAM toward a protumoral M2 phenotype. Stimulation of bone marrow-derived macrophages with lactate was sufficient to induce the expression of the M2-related genes Vegf, Arg1, Relma, Mgl1, and Mgl2. Interestingly, stabilization of HIF- $1 \alpha$ by tumor-derived lactate was the actual driving force for this M2 polarization. Moreover, by inducing Vegf and ARG-1, lactate fulfills a key role in shaping the protumoral phenotype of TAM. While Vegf induces angiogenesis, ARG-1 contributes to tumor growth by the generation of polyamines which act as a proliferative signal for mammalian cancer cells (81). This study proves that nutrients in the tumor microenvironment, such as lactate, can affect the phenotype of infiltrating immune cells, such as TAM.

Research by Penny and colleagues demonstrated that in vitro generated macrophages, cultured with tumor-conditioned media from a pancreatic ductal adanocarcinoma (PDAC) cell line, 


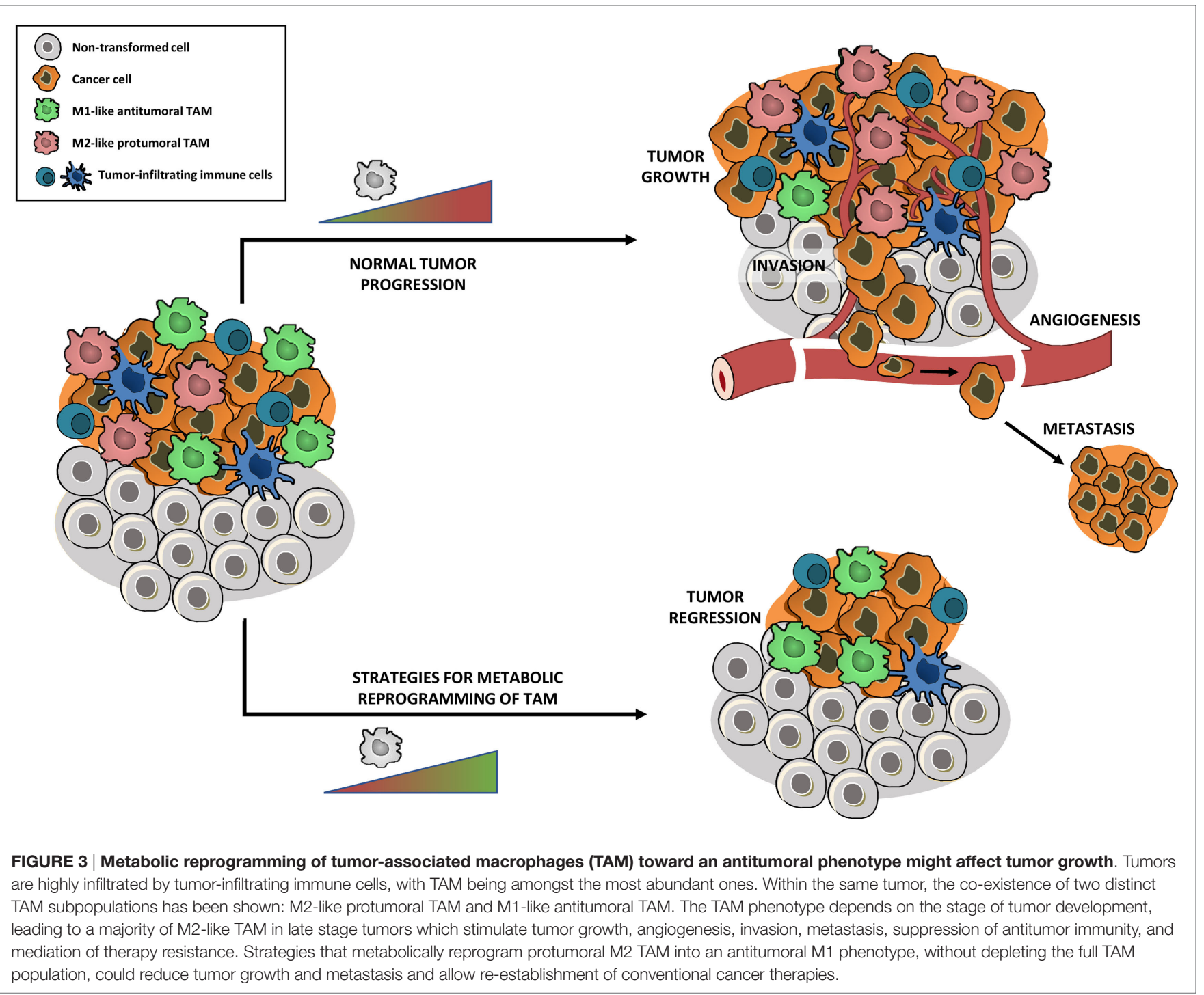

have a pronounced metabolic preference for aerobic glycolysis. In comparison to control macrophages, PDAC TAM showed enhanced angiogenesis and cancer cell extravasation, hence inducing metastasis. Treating PDAC TAM with 2-deoxyglucose, an inhibitor of the first glycolytic enzyme HK2, blocked the pro-metastatic TAM phenotype (82). This study links pancreatic TAM metabolism to aerobic glycolysis, which is in contrast to our current understanding of M2 macrophage metabolism, as extensively described before, claiming their lowered glycolysis and preference for oxidative mitochondrial metabolism.

A recent study by the group of Mazzone revealed that TAM metabolism directly affects tumor vasculature and metastasis, making the link between TAM metabolism and its protumoral functionality. REDD1, an inhibitor of mTOR, is highly expressed by TAM in the hypoxic regions of the tumor, which have been described previously as more M2-like macrophages with high angiogenic potential. Genetic deletion of REDD1 in hypoxic TAM induced mTOR activity, which in turn increased glucose uptake and directed hypoxic macrophage metabolism toward glycolysis. Enhanced glycolysis upon REDD1 deletion caused competition for glucose between hypoxic TAM and tumor endothelial cells. As a result of this competition, tumor vasculature is stabilized, thereby preventing metastasis (83). Thus, any approach diminishing REDD1 activity in TAM could be an interesting strategy to metabolically reprogram TAM toward a M1 phenotype. However, this should be combined with a therapy that allows to specifically target TAM, because REDD1 inhibition will stimulate mTOR signaling and glycolysis in cancer cells and boost their proliferation.

Hence, the challenge for next-level TAM-based antitumor therapies will be to identify metabolic targets that allow repolarizing TAM toward an antitumoral M1 phenotype (Figure 3), without boosting cancer cell metabolism. Therefore, strategies that are able to target TAM metabolism in a specific way will gain much attention. An even more interesting approach would be to look for metabolic targets that allow TAM repolarization and at 
the same time impair cancer cell metabolism. Therefore, drugs that have been shown to disturb cancer cell metabolism could be repurposed to metabolically shift TAM. In this context, drugs that inhibit FAO could be very promising. Several studies indicated the importance of FAO for different types of cancer cells (84). For instance, it has been shown that pharmacological inhibition of FAO impaired proliferation of human leukemia cells (85). On the other hand, blocking FAO in human glioblastoma cells induced oxidative stress and led to bioenergetic failure and cell death (86). A recent study by Schoors and colleagues demonstrated that FAO supports de novo nucleotide synthesis in endothelial cells and subsequently contributes to angiogenesis (87). As described before, FAO is related to the metabolism of IL-4/IL-13 induced M2 macrophages (51). Although it has not been shown that inhibiting FAO shifts M2-like TAM to a M1-like phenotype, impairing FAO could be a promising approach to target protumoral macrophage, endothelial, and cancer cell metabolism at the same time.

The opportunity of targeting immune cell metabolism for cancer therapy is extensively studied in T cells. Potential strategies that aim to alter T-cell metabolism as an effective treatment against cancer recently evolved and have been reviewed in Ref. (88). Interestingly, it was shown that upon PD-1 ligation T-cell metabolism shifts from glycolysis to FAO, encouraging T-cell longevity and impairing their effector function. Hence, currently used immune checkpoint inhibitors, such as PD-1 blocking antibodies, could affect T-cell metabolism by altering glycolysis and subsequently enhance T-cell function (89). Besides immune cells, the metabolism of stromal cells, such as endothelial cells, gained much attention and highlighted interesting therapeutic strategies for cancer therapy $(87,90)$.

\section{Macrophage Polarization beyond Cancer: A Case Study on Obesity and Atherosclerosis}

The concepts concerning macrophage polarization, as discussed above in the context of TAM, can be translated beyond cancer. Also for inflammatory diseases, such as atherosclerosis and obesity, metabolic repolarization of macrophages could be an interesting approach to affect disease outcome.

Atherosclerotic cardiovascular disease is a chronic inflammatory disorder, showing strong macrophage involvement. In case of hypercholesterolemia, apolipoprotein B-containing lipoproteins, such as LDL, accumulate in the arterial wall and are taken up by macrophages. These lipid-laden macrophages, better known as foam cells, lead to plaque formation and secrete inflammatory mediators that persist inflammation and promote plaque progression. Plaque rupture and subsequent formation of blood cloths form the basis for myocardial infarction and stroke, the leading causes of death in western countries $(91,92)$. Although both M1 and M2 macrophages are present in human atherosclerosis, M1 macrophages are the dominant phenotype linked to plaque progression (93). Several studies in mouse models for atherosclerosis demonstrated that inducing M1 polarization of macrophages enhanced disease progression. For example, deletion of the transcription factors NUR77 or KLF4, which both have been shown to drive M2 polarization, induced M1 polarization of macrophages, and enhanced atherosclerosis in apolipoprotein $\mathrm{E}^{-/-}$mice (94, 95). In line with these studies, inducing M2 polarization through administration of the M2-related cytokine IL-13 reduced disease progression (96). Furthermore, lowering lipid levels or enhancing HDL levels in mouse models, induced atherosclerosis regression as a result of a switch toward M2 macrophages in plaques (97, 98). Hence, strategies that allow M2 polarization of macrophages could be beneficial in atherosclerosis disease. A recent study demonstrated that upregulating FAO in macrophages of hypercholesterolemic mice via miR-33 inhibition drives macrophages toward an M2 state and reduces atherosclerosis (99). This study emphasizes how metabolic reprogramming of macrophages can influence disease outcome.

Besides atherosclerosis, the relevance of interfering with macrophage metabolism gained much attention in the context of metabolic diseases, such as obesity. Low-grade systemic chronic inflammation leads to accumulation of macrophages in the adipose tissue of obese humans and mice. While adipose tissue macrophages (ATM) represent less than $10 \%$ of all adipose tissue cells in lean mice and humans, their percentage raises over $50 \%$ in extremely obese mice and near $40 \%$ in obese humans (100). Since macrophages are known as plastic cells that adapt their phenotype to the changing microenvironment, one could expect a switch in the phenotype of ATM during obesity, as described in Ref. (101). Macrophages in lean adipose tissue are considered as anti-inflammatory M2 macrophages, playing a role in maintaining adipose tissue homeostasis by cleaning cellular debris and lipid buffering (uptake and storage of lipids released by adipocytes) (102). Macrophages in obese adipose tissue have been described as pro-inflammatory M1 macrophages and are believed to be the major contributors of obesity-induced insulin resistance, leading to type-2 diabetes, due to the production of pro-inflammatory cytokines, such as TNF (103). More recent papers highlight the importance of the continuous exposure of ATM to lipids in vivo, leading to chronic lipid overloading. $\mathrm{Xu}$ et al. showed that ATM in obese adipose tissue are associated with increased liposomal biogenesis and lipid catabolism (104). More recently, Kratz and colleagues introduced the principle of metabolic activation of ATM, showing that treating macrophages with glucose, insulin, and fatty acids (palmitate) drives the pro-inflammatory ATM phenotype in obese mice (105). Hence, this study indicates that a metabolic trigger can lead to inflammatory macrophage activation, rather than a classic cytokine-driven activation. These new insights imply that interfering with metabolism is a promising approach to dampen the pro-inflammatory ATM phenotype and restore adipose tissue homeostasis in obesity.

Taken together, hyperactivated pro-inflammatory macrophages often contribute to disease progression in inflammatory disorders, such as atherosclerosis and obesity. Therefore, dampening their pro-inflammatory activity is a promising avenue to affect disease outcome. Although, similar to cancer, where repolarization of macrophages toward the pro-inflammatory phenotype has been established as beneficial in inflammatory diseases, such as obesity and atherosclerosis, it would be even more useful to do the opposite and reprogram macrophages toward an 
anti-inflammatory state. Especially in the case of atherosclerosis, forcing macrophages into an anti-inflammatory phenotype has been shown to induce atherosclerosis regression.

\section{CONCLUDING REMARKS}

The growing interest and studies on immunometabolism, in particular, the metabolism of macrophages, raise new therapeutic opportunities to treat inflammatory diseases and cancer. In particular, the metabolic repolarization of macrophages seems to be an interesting approach to fight diseases that show a high macrophage involvement, such as cancer, obesity, and atherosclerosis. Nevertheless, there are some challenges for the future that need to be considered in the study of macrophage metabolism. One of them will be to either only selectively target macrophages or to find metabolic targets that do not affect the disease in a positive way, which is an existing risk in the case of cancer. On the other hand, macrophages should be considered as dynamic cells which adapt their phenotype and possibly also their metabolic state through the different phases of disease, which might hamper metabolic targeting. Furthermore, most of the macrophage metabolism studies have been done in vitro. However, during inflammation or tumor development, macrophages have to deal with a specific microenvironment, characterized by disturbed nutrient and oxygen availability, which instructs their metabolism and

\section{REFERENCES}

1. Pearce EL, Poffenberger MC, Chang CH, Jones RG. Fueling immunity: insights into metabolism and lymphocyte function. Science (2013) 342(6155):1242454. doi:10.1126/science.1242454

2. Ho PC, Liu PS. Metabolic communication in tumors: a new layer of immunoregulation for immune evasion. J Immunother Cancer (2016) 4(1):4. doi:10.1186/s40425-016-0109-1

3. O'Neill LAJ, Kishton RJ, Rathmell J. A guide to immunometabolism for immunologists. Nat Rev Immunol (2016) 16(9):553-65. doi:10.1038/ nri.2016.70

4. O'Neill LA, Pearce EJ. Immunometabolism governs dendritic cell and macrophage function. J Exp Med (2016) 213(1):15-23. doi:10.1084/jem.20151570

5. Mills CD, Thomas AC, Lenz LL, Munder M. Macrophage: SHIP of immunity. Front Immunol (2014) 5:620. doi:10.3389/fimmu.2014.00620

6. Lunt SY, Vander Heiden MG. Aerobic glycolysis: meeting the metabolic requirements of cell proliferation. Annu Rev Cell Dev Biol (2011) 27:441-64. doi:10.1146/annurev-cellbio-092910-154237

7. Warburg O, Wind F, Negelein E. The metabolism of tumors in the body. J Gen Physiol (1927) 8(6):519-30. doi:10.1085/jgp.8.6.519

8. Ghesquière B, Wong BW, Kuchnio A, Carmeliet P. Metabolism of stromal and immune cells in health and disease. Nature (2014) 511(7508):167-76. doi:10.1038/nature13312

9. De Palma M, Lewis CE. Macrophage regulation of tumor responses to anticancer therapies. Cancer Cell (2013) 23(3):277-86. doi:10.1016/j. ccr.2013.02.013

10. Ginhoux F, Schultze JL, Murray PJ, Ochando J, Biswas SK. New insights into the multidimensional concept of macrophage ontogeny, activation and function. Nat Immunol (2016) 17(1):34-40. doi:10.1038/ni.3324

11. Xue J, Schmidt SV, Sander J, Draffehn A, Krebs W, Quester I, et al. Transcriptome-based network analysis reveals a spectrum model of human macrophage activation. Immunity (2014) 40(2):274-88. doi:10.1016/j. immuni.2014.01.006

12. Murray PJ, Allen JE, Biswas SK, Fisher EA, Gilroy DW, Goerdt S, et al. Macrophage activation and polarization: nomenclature and experimental guidelines. Immunity (2014) 41(1):14-20. doi:10.1016/j.immuni.2014.06.008 functionality. Hence, specialized experimental technologies, such as in vivo tracer analysis, will be unconditional to bring macrophage metabolism studies one step further to the in vivo level. We look forward to revolutionary discoveries in the area of macrophage metabolism that will lead to therapeutic targets that could affect disease outcome in inflammatory diseases and cancer.

\section{AUTHOR CONTRIBUTIONS}

XG has contributed in writing, reviewing, and editing the content of this article. EB has contributed in reviewing this article. S-MF and JVG have contributed in writing, reviewing, editing, conceptualization, and supervising this article. All authors have read and approved the manuscript.

\section{FUNDING}

XG is supported by a Ph.D. fellowship of the Research Foundation - Flanders (FWO). EB is supported by a grant from IWT-Vlaanderen. SMF acknowledges funding from Marie Curie-CIG, FWO, KU Leuven-Methusalem Co-Funding, and Bayer Health Care. JVG is supported by several research grants from Vlaamse Liga tegen Kanker, Stichting tegen Kanker, FWOVlaanderen, IWT-Vlaanderen, and Vrije Universiteit Brussel.

13. Mills CD, Harris RA, Ley K. Macrophage polarization: decisions that affect health. JClin Cell Immunol (2015) 6(5):364. doi:10.4172/2155-9899.1000364

14. Mills CD, Kincaid K, Alt JM, Heilman MJ, Hill AM. M-1/M-2 macrophages and the Th1/Th2 paradigm. J Immunol (2000) 164(12):6166-73. doi:10.4049/ jimmunol.164.12.6166

15. Mantovani A, Sica A, Sozzani S, Allavena P, Vecchi A, Locati M. The chemokine system in diverse forms of macrophage activation and polarization. Trends Immunol (2004) 25(12):677-86. doi:10.1016/j.it.2004.09.015

16. Gordon S. Alternative activation of macrophages. Nat Rev Immunol (2003) 3(1):23-35. doi:10.1038/nri978

17. Van Ginderachter JA, Movahedi K, Ghassabeh GH, Meerschaut S, Beschin A, Raes $\mathrm{G}$, et al. Classical and alternative activation of mononuclear phagocytes: picking the best of both worlds for tumor promotion. Immunobiology (2006) 211(6-8):487-501. doi:10.1016/j.imbio.2006.06.002

18. Biswas SK, Mantovani A. Orchestration of metabolism by macrophages. Cell Metab (2012) 15(4):432-7. doi:10.1016/j.cmet.2011.11.013

19. Mills EL, O’Neill LA. Reprogramming mitochondrial metabolism in macrophages as an anti-inflammatory signal. Eur J Immunol (2016) 46(1):13-21. doi:10.1002/eji.201445427

20. Jha AK, Huang SCC, Sergushichev A, Lampropoulou V, Ivanova Y, Loginicheva E, et al. Network integration of parallel metabolic and transcriptional data reveals metabolic modules that regulate macrophage polarization. Immunity (2015) 42(3):419-30. doi:10.1016/j.immuni.2015.02.005

21. Meiser J, Krämer L, Sapcariu SC, Battello N, Ghelfi J, D’Herouel AF, et al. Proinflammatory macrophages sustain pyruvate oxidation through pyruvate dehydrogenase for the synthesis of itaconate and to enable cytokine expression. J Bio Chem (2016) 291(8):3932-46. doi:10.1074/jbc.M115.676817

22. Michelucci A, Cordes T, Ghelfi J, Pailot A, Reiling N, Goldmann O, et al. Immune-responsive gene 1 protein links metabolism to immunity by catalyzing itaconic acid production. Proc Natl Acad Sci US A (2013) 110(19):7820-5. doi: $10.1073 /$ pnas. 1218599110

23. Cordes T, Wallace M, Michelucci A, Divakaruni AS, Sapcariu SC, Sousa $\mathrm{C}$, et al. Immunoresponsive gene 1 and itaconate inhibit succinate dehydrogenase to modulate intracellular succinate levels. J Biol Chem (2016) 291(27):14274-84. doi:10.1074/jbc.M115.685792 
24. Lampropoulou V, Sergushichev A, Bambouskova M, Nair S, Vincent $\mathrm{EE}$, Loginicheva E, et al. Itaconate links inhibition of succinate dehydrogenase with macrophage metabolic remodeling and regulation of inflammation. Cell Metab (2016) 24(1):158-66. doi:10.1016/j. cmet.2016.06.004

25. Tannahill GM, Curtis AM, Adamik J, Palsson-McDermott EM, McGettrick $\mathrm{AF}$, Goel G, et al. Succinate is a danger signal that induces IL- $1 \beta$ via HIF- $1 \alpha$. Nature (2013) 496(7444):238-42. doi:10.1038/nature11986

26. Lorendeau D, Christen S, Rinaldi G, Fendt SM. Metabolic control of signalling pathways and metabolic auto-regulation. Biol Cell (2015) 107(8):251-72. doi:10.1111/boc.201500015

27. Mills EL, Kelly B, Logan A, Costa AS, Varma M, Bryant CE, et al. Succinate dehydrogenase supports metabolic repurposing of mitochondria to drive inflammatory macrophages. Cell (2016) 167(2):457-70. doi:10.1016/j. cell.2016.08.064

28. Moon JS, Hisata S, Park MA, DeNicola GM, Ryter SW, Nakahira K, et al. mTORC1-induced HK1-dependent glycolysis regulates NLRP3 inflammasome activation. Cell Rep (2015) 12:102-15. doi:10.1016/j. celrep.2015.05.046

29. Hayashi M, Sakata M, Takeda T, Yamamoto T, Okamoto Y, Sawada K, et al. Induction of glucose transporter 1 expression through hypoxia-inducible factor $1 \alpha$ under hypoxic conditions in trophoblast-derived cells. J Endocrinol (2004) 183(1):145-54. doi:10.1677/joe.1.05599

30. Obach M, Navarro-Sabaté À, Caro J, Kong X, Duran J, Gómez M, et al. 6-Phosphofructo-2-kinase (pfkfb3) gene promoter contains hypoxia-inducible factor-1 binding sites necessary for transactivation in response to hypoxia. J Biol Chem (2004) 279(51):53562-70. doi:10.1074/jbc.M406096200

31. Ullah MS, Davies AJ, Halestrap AP. The plasma membrane lactate transporter MCT4, but not MCT1, is up-regulated by hypoxia through a HIF-1 $\alpha$ dependent mechanism. J Biol Chem (2006) 281(14):9030-7. doi:10.1074/jbc. M511397200

32. Blouin CC, Pagé EL, Soucy GM, Richard DE. Hypoxic gene activation by lipopolysaccharide in macrophages: implication of hypoxia-inducible factor $1 \alpha$. Blood (2004) 103(3):1124-30. doi:10.1182/blood-2003-07-2427

33. Freemerman AJ, Johnson AR, Sacks GN, Milner JJ, Kirk EL, Troester MA, et al. Metabolic reprogramming of macrophages glucose transporter 1 (GLUT1)-mediated glucose metabolism drives a proinflammatory phenotype. J Biol Chem (2014) 289(11):7884-96. doi:10.1074/jbc.M113.522037

34. Rodríguez-Prados JC, Través PG, Cuenca J, Rico D, Aragonés J, Martín-Sanz P, et al. Substrate fate in activated macrophages: a comparison between innate, classic, and alternative activation. J Immunol (2010) 185(1):605-14. doi:10.4049/jimmunol.0901698

35. Boscá L, González-Ramos S, Prieto P, Fernández-Velasco M, Mojena M, Martín-Sanz P, et al. Metabolic signatures linked to macrophage polarization: from glucose metabolism to oxidative phosphorylation. Biochem Soc Trans (2015) 43(4):740-4. doi:10.1042/BST20150107

36. Tan Z, Xie N, Banerjee S, Cui H, Fu M, Thannickal VJ, et al. The monocarboxylate transporter 4 is required for glycolytic reprogramming and inflammatory response in macrophages. J Biol Chem (2015) 290(1):46-55. doi:10.1074/jbc.M114.603589

37. Qualls JE, Subramanian C, Rafi W, Smith AM, Balouzian L, DeFreitas AA, et al. Sustained generation of nitric oxide and control of mycobacterial infection requires argininosuccinate synthase 1. Cell Host Microbe (2012) 12(3):313-23. doi:10.1016/j.chom.2012.07.012

38. Clementi E, Brown GC, Feelisch M, Moncada S. Persistent inhibition of cell respiration by nitric oxide: crucial role of S-nitrosylation of mitochondrial complex I and protective action of glutathione. Proc Natl Acad Sci U S A (1998) 95(13):7631-6. doi:10.1073/pnas.95.13.7631

39. Kelly B, O’Neill LA. Metabolic reprogramming in macrophages and dendritic cells in innate immunity. Cell Res (2015) 25(7):771-84. doi:10.1038/cr.2015.68

40. Van den Bossche J, Baardman J, Otto NA, van der Velden S, Neele AE, van den Berg SM, et al. Mitochondrial dysfunction prevents repolarization of inflammatory macrophages. Cell Rep (2016) 17(3):684-96. doi:10.1016/j. celrep.2016.09.008

41. Tan HY, Wang N, Li S, Hong M, Wang X, Feng Y. The reactive oxygen species in macrophage polarization: reflecting its dual role in progression and treatment of human diseases. Oxid Med Cell Longev (2016) 2016:16. doi:10.1155/2016/2795090
42. West AP, Brodsky IE, Rahner C, Woo DK, Erdjument-Bromage H, Tempst $\mathrm{P}$, et al. TLR signalling augments macrophage bactericidal activity through mitochondrial ROS. Nature (2011) 472(7344):476-80. doi:10.1038/ nature09973

43. Infantino V, Convertini P, Cucci L, Panaro MA, Di Noia MA, Calvello R, et al. The mitochondrial citrate carrier: a new player in inflammation. Biochem $J$ (2011) 438(3):433-6. doi:10.1042/BJ20111275

44. Infantino V, Iacobazzi V, Palmieri F, Menga A. ATP-citrate lyase is essential for macrophage inflammatory response. Biochem Biophys Res Commun (2013) 440(1):105-11. doi:10.1016/j.bbrc.2013.09.037

45. Infantino V, Iacobazzi V, Menga A, Avantaggiati ML, Palmieri F. A key role of the mitochondrial citrate carrier (SLC25A1) in TNFo-and IFN $\gamma$-triggered inflammation. Biochim Biophys Acta (2014) 1839(11):1217-25. doi:10.1016/j. bbagrm.2014.07.013

46. Baardman J, Licht I, de Winther MP, Van den Bossche J. Metabolic-epigenetic crosstalk in macrophage activation. Epigenomics (2015) 7(7):1155-64. doi:10.2217/epi.15.71

47. Wellen KE, Hatzivassiliou G, Sachdeva UM, Bui TV, Cross JR, Thompson CB. ATP-citrate lyase links cellular metabolism to histone acetylation. Science (2009) 324(5930):1076-80. doi:10.1126/science.1164097

48. Feingold KR, Shigenaga JK, Kazemi MR, McDonald CM, Patzek SM, Cross AS, et al. Mechanisms of triglyceride accumulation in activated macrophages. J Leukocyte Biol (2012) 92(4):829-39. doi:10.1189/jlb.1111537

49. Ecker J, Liebisch G, Englmaier M, Grandl M, Robenek H, Schmitz G. Induction of fatty acid synthesis is a key requirement for phagocytic differentiation of human monocytes. Proc Natl Acad Sci U S A (2010) 107(17):7817-22. doi:10.1073/pnas.0912059107

50. Galván-Peña S, O’Neill LA. Metabolic reprograming in macrophage polarization. Front Immunol (2014) 5:420. doi:10.3389/fimmu.2014.00420

51. Vats D, Mukundan L, Odegaard JI, Zhang L, Smith KL, Morel CR, et al. Oxidative metabolism and PGC- $1 \beta$ attenuate macrophage-mediated inflammation. Cell Metab (2006) 4(1):13-24. doi:10.1016/j.cmet.2006.05.011

52. Huang SC, Everts B, Ivanova Y, O'Sullivan D, Nascimento M, Smith AM, et al. Cell-intrinsic lysosomal lipolysis is essential for alternative activation of macrophages. Nat Immunol (2014) 15(9):846-55. doi:10.1038/ni.2956

53. Malandrino MI, Fucho R, Weber M, Calderon-Dominguez M, Mir JF, Valcarcel L, et al. Enhanced fatty acid oxidation in adipocytes and macrophages reduces lipid-induced triglyceride accumulation and inflammation. Am J Physiol Endocrinol Metab (2015) 308(9):756-69. doi:10.1152/ ajpendo.00362.2014

54. Nomura M, Liu J, Rovira II, Gonzalez-Hurtado E, Lee J, Wolfgang MJ, et al. Fatty acid oxidation in macrophage polarization. Nat Immunol (2016) 17(3):216-7. doi:10.1038/ni.3366

55. Chawla A. Control of macrophage activation and function by PPARs. Circ Res (2010) 106(10):1559-69. doi:10.1161/CIRCRESAHA.110.216523

56. Odegaard JI, Ricardo-Gonzalez RR, Goforth MH, Morel CR, Subramanian V, Mukundan L, et al. Macrophage-specific PPARgamma controls alternative activation and improves insulin resistance. Nature (2007) 447(7148):1116-20. doi:10.1038/nature05894

57. Coste A, Dubourdeau M, Linas MD, Cassaing S, Lepert JC, Balard P, et al. $\mathrm{PPAR} \gamma$ promotes mannose receptor gene expression in murine macrophages and contributes to the induction of this receptor by IL-13. Immunity (2003) 19(3):329-39. doi:10.1016/S1074-7613(03)00229-2

58. Rath M, Müller I, Kropf P, Closs EI, Munder M. Metabolism via arginase or nitric oxide synthase: two competing arginine pathways in macrophages. Front Immunol (2014) 5:532. doi:10.3389/fimmu.2014.00532

59. Modolell M, Corraliza IM, Link F, Soler G, Eichmann K. Reciprocal regulation of the nitric oxide synthase/arginase balance in mouse bone marrow-derived macrophages by TH 1 and TH 2 cytokines. Eur J Immunol (1995) 25(4):1101-4. doi:10.1002/eji.1830250436

60. Pauleau AL, Rutschman R, Lang R, Pernis A, Watowich SS, Murray PJ. Enhancer-mediated control of macrophage-specific arginase I expression. J Immunol (2004) 172(12):7565-73. doi:10.4049/jimmunol.172.12.7565

61. Gallardo-Soler A, Gómez-Nieto C, Campo ML, Marathe C, Tontonoz P, Castrillo A, et al. Arginase I induction by modified lipoproteins in macrophages: a peroxisome proliferator-activated receptor- $\gamma / \mathrm{d}$-mediated effect that links lipid metabolism and immunity. Mol Endocrinol (2008) 22(6):1394-402. doi:10.1210/me.2007-0525 
62. Hardbower DM, Asim M, Luis PB, Singh K, Barry DP, Yang C, et al. Ornithine decarboxylase regulates M1 macrophage activation and mucosal inflammation via histone modifications. Proc Natl Acad Sci U S A (2017) 114(5):E751-60. doi:10.1073/pnas.1614958114

63. Haschemi A, Kosma P, Gille L, Evans CR, Burant CF, Starkl P, et al. The sedoheptulose kinase CARKL directs macrophage polarization through control of glucose metabolism. Cell Metab (2012) 15(6):813-26. doi:10.1016/j. cmet.2012.04.023

64. Lahmar Q, Keirsse J, Laoui D, Movahedi K, Van Overmeire E, Van Ginderachter JA. Tissue-resident versus monocyte-derived macrophages in the tumor microenvironment. Biochim Biophys Acta (2016) 1865(1):23-34. doi:10.1016/j.bbcan.2015.06.009

65. Tennant DA, Durán RV, Gottlieb E. Targeting metabolic transformation for cancer therapy. Nat Rev Cancer (2010) 10(4):267-77. doi:10.1038/nrc2817

66. Elia I, Schmieder R, Christen S, Fendt SM. Organ-specific cancer metabolism and its potential for therapy. Handb Exp Pharmacol (2016) 233:321-53. doi:10.1007/164_2015_10

67. Zhang QW, Liu L, Gong CY, Shi HS, Zeng YH, Wang XZ, et al. Prognostic significance of tumor-associated macrophages in solid tumor: a meta-analysis of the literature. PLoS One (2012) 7(12):e50946. doi:10.1371/journal. pone.0050946

68. Qian BZ, Pollard JW. Macrophage diversity enhances tumor progression and metastasis. Cell (2010) 141(1):39-51. doi:10.1016/j.cell.2010.03.014

69. Movahedi K, Laoui D, Gysemans C, Baeten M, Stangé G, Van den Bossche $J$, et al. Different tumor microenvironments contain functionally distinct subsets of macrophages derived from Ly6C (high) monocytes. Cancer Res (2010) 70(14):5728-39. doi:10.1158/0008-5472.CAN-09-4672

70. Laoui D, Van Overmeire E, Di Conza G, Aldeni C, Keirsse J, Morias Y, et al. Tumor hypoxia does not drive differentiation of tumor-associated macrophages but rather fine-tunes the M2-like macrophage population. Cancer Res (2014) 74(1):24-30. doi:10.1158/0008-5472.CAN-13-1196

71. Van Overmeire E, Stijlemans B, Heymann F, Keirsse J, Morias Y, Elkrim Y, et al. M-CSF and GM-CSF receptor signaling differentially regulate monocyte maturation and macrophage polarization in the tumor microenvironment. Cancer Res (2016) 76(1):35-42. doi:10.1158/0008-5472.CAN-15-0869

72. Biswas SK, Sica A, Lewis CE. Plasticity of macrophage function during tumor progression: regulation by distinct molecular mechanisms. J Immunol (2008) 180(4):2011-7. doi:10.4049/jimmunol.180.4.2011

73. Mantovani A, Allavena P. The interaction of anticancer therapies with tumor-associated macrophages. JExp Med (2015) 212(4):435-45. doi:10.1084/jem.20150295

74. Loberg RD, Ying C, Craig M, Day LL, Sargent E, Neeley C, et al. Targeting CCL2 with systemic delivery of neutralizing antibodies induces prostate cancer tumor regression in vivo. Cancer Res (2007) 67(19):9417-24. doi:10.1158/0008-5472.CAN-07-1286

75. Sanford DE, Belt BA, Panni RZ, Mayer A, Deshpande AD, Carpenter D, et al. Inflammatory monocyte mobilization decreases patient survival in pancreatic cancer: a role for targeting the CCL2/CCR2 axis. Clin Cancer Res (2013) 19(13):3404-15. doi:10.1158/1078-0432.CCR-13-0525

76. Sandhu SK, Papadopoulos K, Fong PC, Patnaik A, Messiou C, Olmos D, et al. A first-in-human, first-in-class, phase I study of carlumab (CNTO 888), a human monoclonal antibody against CC-chemokine ligand 2 in patients with solid tumors. Cancer Chemother Pharmacol (2013) 71(4):1041-50. doi:10.1007/s00280-013-2099-8

77. Bonapace L, Coissieux MM, Wyckoff J, Mertz KD, Varga Z, Junt T, et al. Cessation of CCL2 inhibition accelerates breast cancer metastasis by promoting angiogenesis. Nature (2014) 515(7525):130-3. doi:10.1038/nature13862

78. Engblom C, Pfirschke C, Pittet MJ. The role of myeloid cells in cancer therapies. Nat Rev Cancer (2016) 16(7):447-62. doi:10.1038/nrc.2016.54

79. Casazza A, Laoui D, Wenes M, Rizzolio S, Bassani N, Mambretti M, et al. Impeding macrophage entry into hypoxic tumor areas by Sema3A/Nrp1 signaling blockade inhibits angiogenesis and restores antitumor immunity. Cancer Cell (2013) 24(6):695-709. doi:10.1016/j.ccr.2013.11.007

80. Pyonteck SM, Akkari L, Schuhmacher AJ, Bowman RL, Sevenich L, Quail DF, et al. CSF-1R inhibition alters macrophage polarization and blocks glioma progression. Nat Med (2013) 19(10):1264-72. doi:10.1038/nm.3337

81. Colegio OR, Chu NQ, Szabo AL, Chu T, Rhebergen AM, Jairam V, et al. Functional polarization of tumour-associated macrophages by tumour-derived lactic acid. Nature (2014) 513(7519):559-63. doi:10.1038/ nature 13490

82. Penny HL, Sieow JL, Adriani G, Yeap WH, See Chi Ee P, San Luis B, et al. Warburg metabolism in tumor-conditioned macrophages promotes metastasis in human pancreatic ductal adenocarcinoma. Oncoimmunology (2016) 5(8):e1191731. doi:10.1080/2162402X 2016.1191731

83. Wenes M, Shang M, Di Matteo M, Goveia J, Martín-Pérez R, Serneels J, et al. Macrophage metabolism controls tumor blood vessel morphogenesis and metastasis. Cell Metab (2016) 24(5):701-15. doi:10.1016/j.cmet.2016.09.008

84. Currie E, Schulze A, Zechner R, Walther TC, Farese RV Jr. Cellular fatty acid metabolism and cancer. Cell Metab (2013) 18(2):153-61. doi:10.1016/j. cmet.2013.05.017

85. Samudio I, Harmancey R, Fiegl M, Kantarjian H, Konopleva M, Korchin $\mathrm{B}$, et al. Pharmacologic inhibition of fatty acid oxidation sensitizes human leukemia cells to apoptosis induction. JClin Invest (2010) 120(1):142-56. doi:10.1172/JCI38942

86. Pike LS, Smift AL, Croteau NJ, Ferrick DA, Wu M. Inhibition of fatty acid oxidation by etomoxir impairs NADPH production and increases reactive oxygen species resulting in ATP depletion and cell death in human glioblastoma cells. Biochim Biophys Acta (2011) 1807(6):726-34. doi:10.1016/j. bbabio.2010.10.022

87. Schoors S, Bruning U, Missiaen R, Queiroz KC, Borgers G, Elia I, et al. Fatty acid carbon is essential for dNTP synthesis in endothelial cells. Nature (2015) 520(7546):192-7. doi:10.1038/nature14362

88. Chang C-H, Pearce EL. Emerging concepts in immunotherapy - T cell metabolism as a therapeutic target. Nat Immunol (2016) 17(4):364-8. doi:10.1038/ni.3415

89. Patsoukis N, Bardhan K, Chatterjee P, Sari D, Liu B, Bell LN, et al. PD-1 alters T-cell metabolic reprogramming by inhibiting glycolysis and promoting lipolysis and fatty acid oxidation. Nat Commun (2015) 6:6692. doi:10.1038/ ncomms7692

90. Verdegem D, Moens S, Stapor P, Carmeliet P. Endothelial cell metabolism: parallels and divergences with cancer cell metabolism. Cancer Metab (2014) 2:19. doi:10.1186/2049-3002-2-19

91. Moore KJ, Sheedy FJ, Fisher EA. Macrophages in atherosclerosis: a dynamic balance. Nat Rev Immunol (2013) 13(10):709-21. doi:10.1038/nri3520

92. Peled M, Fisher EA. Dynamic aspects of macrophage polarization during atherosclerosis progression and regression. Front Immunol (2014) 5:579. doi:10.3389/fimmu.2014.00579

93. Stöger JL, Gijbels MJ, van der Velden S, Manca M, van der Loos CM, Biessen EA, et al. Distribution of macrophage polarization markers in human atherosclerosis. Atherosclerosis (2012) 225(2):461-8. doi:10.1016/j. atherosclerosis.2012.09.013

94. Hanna RN, Shaked I, Hubbeling HG, Punt JA, Wu R, Herrley E, et al. NR4A1 (Nur77) deletion polarizes macrophages toward an inflammatory phenotype and increases atherosclerosis. Circ Res (2012) 110(3):416-27. doi:10.1161/ CIRCRESAHA.111.253377

95. Sharma N, Lu Y, Zhou G, Liao X, Kapil P, Anand P, et al. Myeloid Krüppellike factor 4 deficiency augments atherogenesis in ApoE-/- mice-brief report. Arterioscler Thromb Vasc Biol (2012) 32(12):2836-8. doi:10.1161/ ATVBAHA.112.300471

96. Cardilo-Reis L, Gruber S, Schreier SM, Drechsler M, Papac-Milicevic N, Weber $\mathrm{C}$, et al. Interleukin-13 protects from atherosclerosis and modulates plaque composition by skewing the macrophage phenotype. EMBO Mol Med (2012) 4(10):1072-86. doi:10.1002/emmm.201201374

97. Feig JE, Parathath S, Rong JX, Mick SL, Vengrenyuk Y, Grauer L, et al. Reversal of hyperlipidemia with a genetic switch favorably affects the content and inflammatory state of macrophages in atherosclerotic plaques. Circulation (2011) 123(9):989-98. doi:10.1161/CIRCULATIONAHA.110.984146

98. Feig JE, Rong JX, Shamir R, Sanson M, Vengrenyuk Y, Liu J, et al. HDL promotes rapid atherosclerosis regression in mice and alters inflammatory properties of plaque monocyte-derived cells. Proc Natl Acad Sci U S A (2011) 108(17):7166-71. doi:10.1073/pnas.1016086108

99. Ouimet M, Ediriweera HN, Gundra UM, Sheedy FJ, Ramkhelawon B, Hutchison SB, et al. microRNA-33-dependent regulation of macrophage metabolism directs immune cell polarization in atherosclerosis. J Clin Invest (2015) 125(12):4334-48. doi:10.1172/JCI81676 
100. Weisberg SP, McCann D, Desai M, Rosenbaum M, Leibel RL, Ferrante AW Jr. Obesity is associated with macrophage accumulation in adipose tissue. J Clin Invest (2003) 112(12):1796-808. doi:10.1172/JCI19246

101. Lumeng CN, Bodzin JL, Saltiel AR. Obesity induces a phenotypic switch in adipose tissue macrophage polarization. J Clin Invest (2007) 117(1):175-84. doi:10.1172/JCI29881

102. Kosteli A, Sugaru E, Haemmerle G, Martin JF, Lei J, Zechner R, et al. Weight loss and lipolysis promote a dynamic immune response in murine adipose tissue. J Clin Invest (2010) 120(10):3466-79. doi:10.1172/JCI42845

103. Uysal KT, Wiesbrock SM, Marino MW, Hotamisligil GS. Protection from obesity-induced insulin resistance in mice lacking TNF- $\alpha$ function. Nature (1997) 389(6651):610-4. doi:10.1038/39335

104. Xu X, Grijalva A, Skowronski A, van Eijk M, Serlie MJ, Ferrante AW Jr. Obesity activates a program of lysosomal-dependent lipid metabolism in adipose tissue macrophages independently of classic activation. Cell Metab (2013) 18(6):816-30. doi:10.1016/j.cmet.2013.11.001
105. Kratz M, Coats BR, Hisert KB, Hagman D, Mutskov V, Peris E, et al. Metabolic dysfunction drives a mechanistically distinct proinflammatory phenotype in adipose tissue macrophages. Cell Metab (2014) 20(4):614-25. doi:10.1016/j. cmet.2014.08.010

Conflict of Interest Statement: The authors declare that the research was conducted in the absence of any commercial or financial relationships that could be construed as a potential conflict of interest.

Copyright (C) 2017 Geeraerts, Bolli, Fendt and Van Ginderachter. This is an open-access article distributed under the terms of the Creative Commons Attribution License (CC BY). The use, distribution or reproduction in other forums is permitted, provided the original author(s) or licensor are credited and that the original publication in this journal is cited, in accordance with accepted academic practice. No use, distribution or reproduction is permitted which does not comply with these terms. 